PROCEEDINGS OF THE

AMERICAN MATHEMATICAL SOCIETY

Volume 128, Number 2, Pages 387-396

$\mathrm{S}$ 0002-9939(99)05298-3

Article electronically published on July 6, 1999

\title{
LOG-POLYNOMIAL PERIOD FUNCTIONS FOR NONDISCRETE HECKE GROUPS
}

\author{
ABDULKADIR HASSEN
}

(Communicated by Dennis A. Hejhal)

\begin{abstract}
Existence of automorphic integrals associated with nondiscrete Hecke groups will be considered. Multiplier systems for some of these groups will be discussed.
\end{abstract}

\section{INTRODUCTION}

Let $\lambda>0$. The Hecke group $G(\lambda)$ is the group generated by

$$
S_{\lambda}=\left(\begin{array}{cc}
1 & \lambda \\
0 & 1
\end{array}\right) \quad \text { and } \quad T=\left(\begin{array}{cc}
0 & -1 \\
1 & 0
\end{array}\right) .
$$

$G(\lambda)$ acts on $\mathcal{H}$ by $M z=\frac{a z+b}{c z+d}$, where $M=\left(\begin{array}{ll}a & b \\ c & d\end{array}\right) \in G(\lambda)$ and $z \in \mathcal{H}$. In this case, we identify $M$ with its negative $-M$ and consider the elements of $G(\lambda)$ as fractional linear transformations.

Marvin Knopp in [6] and Knopp and Mark Sheingorn in [7] showed that there are infinitely many linearly independent Dirichlet series with preassigned poles of a given order which satisfy functional equations for the theta group $G(2)$. This shows that in Hamburger's Theorem (see [2]) one cannot relax the assumption about the pole of the Dirichlet series to arrive at the conclusion of the uniqueness of the Riemann zeta function. In [2] the author showed that for the full modular group the only Dirichlet series with functional equation are those associated with modular forms. Also, for many of the discrete Hecke groups similar results were obtained. These results were obtained by applying Hecke's Lemma, which enables one to switch from Dirichlet series with functional equations to automorphic integrals with log-polynomial period functions.

The question we consider in this article is the following. If there is a nontrivial automorphic integral associated with a Hecke group, is the group discrete?

It is well-known (see [1] and [3]) that the only discrete Hecke groups are those for which

$$
\lambda \geq 2 \quad \text { or } \quad \lambda=2 \cos (\pi / p), p \in \mathbf{Z}, p \geq 3 .
$$

Thus we shall be dealing with Hecke groups for which $\lambda=2 \cos (\theta \pi), \quad 0<\theta<$ $1 / 2, \quad \theta \neq 1 / p, \quad p \in \mathbf{Z}$.

Received by the editors April 10, 1998 .

1991 Mathematics Subject Classification. Primary 11F66.

Key words and phrases. Dirichlet series with functional equation, automorphic integrals, Hecke groups. 
In Section 2, we give the definitions of multiplier systems for Hecke groups and give a generalization of a characterization of multiplier systems given in [2]. We also define automorphic integrals and log-polynomial period functions. In Section 3 , we deal with the case when $\theta$ is rational. In this case, we will show that the groups admit an elliptic element of finite order $\neq 2$ and apply the methods used in [2] to show that the nondiscrete groups of this type do not support automorphic integrals.

We close this section by stating Hecke's Lemma as generalized by Weil in [9].

Lemma 1.1 (Riemann-Hecke-Bochner-Weil Correspondence). Let $f$ and $g$ be continuous functions on $(0, \infty)$ such that

$$
\begin{gathered}
f(y), g(1 / y)=\mathcal{O}\left(e^{-a y}\right), \quad y \rightarrow \infty, \quad a>0, \\
f(y), g(1 / y)=\mathcal{O}\left(y^{-b}\right), \quad y \rightarrow 0, \quad b>0 .
\end{gathered}
$$

Throughout, we shall write $s=\sigma+i t$ where $\sigma$ and $t$ are real numbers. Define

$$
\Phi(s)=\int_{0}^{\infty} f(y) y^{s-1} d y \quad \text { and } \quad \Psi(s)=\int_{0}^{\infty} g(y) y^{s-1} d y .
$$

Assume that for some $\sigma_{0}>b, \sigma_{0}^{\prime}<-b$,

$$
\Phi\left(\sigma_{0}+i t\right)=\mathcal{O}\left(|t|^{-2}\right) \quad \text { and } \quad \Psi\left(\sigma_{0}^{\prime}+i t\right)=\mathcal{O}\left(|t|^{-2}\right) \text { as }|t| \rightarrow \infty .
$$

Let $Q(s)$ be a rational function which vanishes at infinity and let $s_{1}, \cdots, s_{m}$ be the poles of $Q$. Assume that $\sigma_{0}^{\prime} \leq \operatorname{Re}\left(s_{\nu}\right) \leq \sigma_{0}$ for each $\nu$. Then the following are equivalent:

(A) $\quad f(y)-g(y)=\sum_{\nu=1}^{m} \operatorname{Res}\left(Q(s) y^{-s}, s_{\nu}\right)$;

$(B) \quad \Phi(s)-Q(s)$ and $\Psi(s)-Q(s)$ can be continued to the same entire function $F(s)$ which is bounded in every vertical strip.

Here Res $\left(Q(s) y^{-s}, s_{\nu}\right)$ is the residue of $Q(s) y^{-s}$ at $s_{\nu}$.

Remark 1.1. Let $Q(z)=\sum_{\nu=1}^{m}\left\{\frac{b(\nu, 0)}{\left(z-s_{\nu}\right)^{n_{\nu}}}+\cdots+\frac{b\left(\nu, n_{\nu}-1\right)}{\left(z-s_{\nu}\right)}\right\}$ be a rational function with $b(\nu, 0) \neq 0$. Then it can easily be seen that

(1) $\sum_{\nu=1}^{m} \operatorname{Res}\left(Q(z) y^{-z}, s_{\nu}\right)=\sum_{\nu=1}^{m} \sum_{j=0}^{n_{\nu}-1} \frac{(-1)^{j}}{j !} b\left(\nu, n_{\nu}-j-1\right)(\log y)^{j} y^{-s_{\nu}}$.

We apply the following corollary of Hecke's Lemma in the case when $\lambda_{1}=\lambda_{2}=\lambda$ and $G=\bar{v}(T) F$.

Corollary 1.1. Let $\lambda_{1}, \lambda_{2}>0, \quad k$ be a real number, $\gamma_{j}$ and $c(j, t)$ be complex numbers and let $t, j, m$, and $n_{j}$ be nonnegative integers. Let $\left\{a_{n}\right\}$ and $\left\{b_{n}\right\}$ be sequences of complex numbers such that

$$
a_{n}, b_{n}=\mathcal{O}\left(n^{c}\right) \text { as } n \rightarrow \infty, \quad \text { for some } c>0 .
$$

Define

$$
\begin{array}{cc}
\phi(s)=\sum_{n=1}^{\infty} a_{n} n^{-s}, & \psi(s)=\sum_{n=1}^{\infty} b_{n} n^{-s} \\
\Phi(s)=\left(\frac{2 \pi}{\lambda_{1}}\right)^{-s} \Gamma(s) \phi(s), & \Psi(s)=\left(\frac{2 \pi}{\lambda_{2}}\right)^{-s} \Gamma(s) \psi(s) ; \\
F(z)=\sum_{n=0}^{\infty} a_{n} e^{2 \pi i z / \lambda_{1}}, & G(z)=\sum_{n=0}^{\infty} b_{n} e^{2 \pi i z / \lambda_{2}}
\end{array}
$$


$Q(s)=\sum_{j=1}^{m} \sum_{t=0}^{n_{j}} \frac{d(j, t)}{\left(s-\gamma_{j}\right)^{n_{j}-t+1}}-\frac{a_{0}}{s}+\frac{e^{-\pi i k} b_{0}}{s-2 k}, \quad q(z)=\sum_{j=1}^{m} \sum_{t=0}^{n_{j}-1} c(j, t)(\log z)^{t} z^{\gamma_{j}}$,

where $c(j, t)=\frac{(-1)^{t}}{t !} d\left(j, n_{j}-t-1\right)$. Then the following are equivalent:

(A) $\quad z^{-2 k} G(-1 / z)=F(z)+q(z)$;

$(B) \quad \Phi(s)-Q(s)$ and $\Psi(s)-Q(s)$ can be continued to the same entire function which is bounded in every lacunary vertical strip:

$$
\sigma_{1} \leq \sigma \leq \sigma_{2}, \quad|\operatorname{Im}(s)| \geq t_{0}>0
$$

and they satisfy the functional equation

$$
\Phi(s)=e^{-\pi i k} \Psi(2 k-s) .
$$

\section{Multiplier Systems for the NONDiscrete HeCKe Groups}

In what follows, $k$ will be a fixed real number. We shall denote by $\mathbf{Z}, \mathbf{C}$, and $\mathcal{H}$ the set of integers, the set of complex numbers, and the upper half-plane, respectively. For $z \in \mathbf{C}$, we assume that $-\pi \leq \arg z<\pi$ and define

$$
(c z+d)^{r}=|c z+d|^{r} \exp \{r i \arg (c z+d)\},
$$

where $r, c, d$ are real numbers.

A multiplier system in weight $2 k$ for $G(\lambda)$ is a complex-valued function $v$ defined on $G(\lambda)$ such that the following two properties hold:

$$
\begin{gathered}
|v(M)|=1 \quad \forall M \in G(\lambda), \\
v\left(M_{3}\right)\left(c_{3} z+d_{3}\right)^{2 k}=v\left(M_{1}\right) v\left(M_{2}\right)\left(c_{1} M_{2} z+d_{1}\right)^{2 k}\left(c_{2} z+d_{2}\right)^{2 k},
\end{gathered}
$$

for all $M_{1}, M_{2} \in G(\lambda)$, with $M_{3}=M_{1} M_{2}, M_{j}=\left(\begin{array}{cc}a_{j} & b_{j} \\ c_{j} & d_{j}\end{array}\right), j=1,2,3$, and $\forall z \in \mathcal{H}$.

Remark 2.1. (i) Condition (4) is sometimes called the consistency condition.

(ii) By taking $M_{1}=M_{2}=I$ in (4), we get $v(I)=v(I)^{2}$ and by (3) we conclude that $v(I)=1$. If we take $M_{1}=M_{2}=-I$ in (4), we get $v(-I)(-1)^{2 k}= \pm 1$. It has been shown in [2] that the only multiplier systems of significance are those for which $v(-I)(-1)^{2 k}=1$ and hence we shall make this assumption for the remainder of our discussion.

(iii) We define $v\left(S_{\lambda}\right)=e^{2 \pi i \kappa}, 0 \leq \kappa<1$. We record the following observations for future reference:
(a) $v(T)= \pm e^{-\pi i k}$
(b) $v\left(T S_{\lambda}^{-1} T\right)=e^{2 \pi i(k-\kappa)}$,
(c) $v\left(-T S_{\lambda}^{-1} T\right)=e^{-2 \pi i \kappa}$.

Lemma 2.1. Suppose $\lambda$ is given by

$$
\lambda=2 \cos (\pi r / p), r, p \in \mathbf{Z},(r, p)=1,1<r<\frac{p}{2} .
$$

Let $v$ be a multiplier system in weight $2 k$ for $G(\lambda)$.

(i) If $v(T)=e^{-\pi i k}$ or $p$ is even, then $(p-2 r) k-2 p \kappa$ is an even integer.

(ii) If $v(T)=-e^{-\pi i k}$ and $p$ is odd, then $(p-2 r) k-2 p \kappa$ is an odd integer. 
Proof. First we show that there are two relations among the generators $S_{\lambda}$ and $T$ of $G(\lambda)$; namely,

$$
T^{2}=-I \quad \text { and } \quad\left(S_{\lambda} T\right)^{p}=(-1)^{r} I .
$$

The first part of (6) is obvious. Let $V_{n}=\left(S_{\lambda} T\right)^{n}=\left(\begin{array}{ll}a_{n} & b_{n} \\ c_{n} & d_{n}\end{array}\right)$. By induction we can show that

$$
a_{n}=\frac{\sin \frac{(n+1) r \pi}{p}}{\sin \frac{r \pi}{p}}, b_{n}=-\frac{\sin \frac{n r \pi}{p}}{\sin \frac{r \pi}{p}}, c_{n}=\frac{\sin \frac{n r \pi}{p}}{\sin \frac{r \pi}{p}}, d_{n}=-\frac{\sin \frac{(n-1) r \pi}{p}}{\sin \frac{r \pi}{p}}
$$

The second part of (6) follows from this. Then by (6), $V_{p}=(-1)^{r} I=\left(S_{\lambda} T\right)^{p}$ and for any $z \in \mathcal{H}$, we have

$$
\begin{aligned}
1 & =v(I)=v(-I)(-1)^{2 k}=v\left(V_{p}\right)(-1)^{2 k} \\
& =v\left(V_{1}\right) v\left(V_{p-1}\right)\left(c_{1} V_{p-1} z+d_{1}\right)^{2 k}\left(c_{p-1} z+d_{p-1}\right)^{2 k} \\
& =v\left(V_{1}\right) v\left(V_{p-1}\right)\left(V_{p-1} z\right)^{2 k}\left(c_{p-1} z+d_{p-1}\right)^{2 k},
\end{aligned}
$$

since $V_{1}=S_{\lambda} T=\left(\begin{array}{cc}\lambda & -1 \\ 1 & 0\end{array}\right)$ and hence $c_{1}=1$ and $d_{1}=0$. But $V_{p-1}=V_{1} V_{p-2}$. Hence we have

$$
\begin{aligned}
v\left(V_{p-1}\right)\left(c_{p-1} z+d_{p-1}\right)^{2 k} & =v\left(V_{1}\right) v\left(V_{p-1}\right)\left(c_{1} V_{p-2} z+d_{1}\right)^{2 k}\left(c_{p-2} z+d_{p-2}\right)^{2 k} \\
& =v\left(V_{1}\right) v\left(V_{p-2}\right)\left(V_{p-2} z\right)^{2 k}\left(c_{p-2} z+d_{p-2}\right)^{2 k} .
\end{aligned}
$$

Thus

$$
1=v\left(V_{1}\right)^{2}\left(V_{p-1} z\right)^{2 k}\left(V_{p-2} z\right)^{2 k} v\left(V_{p-2}\right)\left(c_{p-2} z+d_{p-2}\right)^{2 k} .
$$

Proceeding inductively, we obtain

$$
1=v\left(V_{1}\right)^{p}\left(V_{p-1} z\right)^{2 k}\left(V_{p-2} z\right)^{2 k} \cdots\left(V_{1} z\right)^{2 k} z^{2 k} .
$$

If we put $z=e^{\frac{r \pi i}{p}}$, then we have $V_{p-1} z=V_{p-2} z=\cdots=V_{1} z=z=e^{\frac{r \pi i}{p}}$. Substituting this in (8) and observing the fact that $v\left(V_{1}\right)=v\left(S_{\lambda} T\right)=v\left(S_{\lambda}\right) v(T)=$ $e^{2 \pi i \kappa} v(T)$, we get

$$
v(T)^{p} e^{2 \pi i p \kappa} e^{2 \pi i r k}=1 .
$$

(i) If $v(T)=e^{-\pi i k}$ or $p$ is an even integer, then $v(T)^{p}=e^{-\pi i k p}$ and (9) yields $e^{-\pi i p k+2 \pi i p \kappa+2 \pi i r k}=1$; that is, $(p-2 r) k-2 p \kappa$ is an even integer.

(ii) If $v(T)=-e^{-\pi i k}$ and $p$ is an odd integer, then $v(T)^{p}=-e^{-\pi i k p}$ and (9) gives $e^{-\pi i p k+2 \pi i p \kappa+2 \pi i r k}=-1$; that is, $(p-2 r) k-2 p \kappa$ is an odd integer. This completes the proof.

This lemma generalizes Lemma 2.2 of [2].

A log-polynomial sum is a function of the form

$$
q(z)=\sum_{j=0}^{n} z^{\gamma_{j}} \sum_{t=0}^{m_{j}} c(j, t)(\log z)^{t},
$$

where $\gamma_{1}, \ldots, \gamma_{n}$ and the coefficients $c(j, t)$ are complex numbers, and $n, j, m_{j}$ and $t$ are nonnegative integers. Here $z^{\alpha}$ is defined by $e^{\alpha \log z}$, where $\log z$ is the principal branch of the logarithm function. 
A log-polynomial sum $q(z)$ is said to be a log-polynomial period function (LPPF) of weight $2 k$ and multiplier system $v$ for the Hecke group $G(\lambda)$, if there exists a function $F$ defined and holomorphic in $\mathcal{H}$ such that:

$$
\begin{gathered}
e^{-2 \pi i \kappa} F(z+\lambda)=F(z), \\
\bar{v}(T) z^{-2 k} F\left(-\frac{1}{z}\right)=F(z)+q(z),
\end{gathered}
$$

where $e^{2 \pi i \kappa}=v\left(S_{\lambda}\right)$.

A function $F$ satisfying (11) and (12) is called an automorphic integral of weight $2 k$ and multiplier system $v$ for $G(\lambda)$, if it has an exponential series expansion:

$$
F(z)=\sum_{n=0}^{\infty} a_{n} e^{2 \pi i(n+\kappa) z / \lambda},
$$

where $a_{n} \in \mathbf{C}$ and satisfy the growth condition

$$
a_{n}=\mathcal{O}\left(n^{c}\right) \quad \text { as } n \rightarrow \infty, \quad c>0 .
$$

In this case we say that $q$ is the log-polynomial period function of the automorphic integral $F$.

If we use the slash operator $\left.\right|_{v} ^{2 k}$ defined by

$$
\left(F \mid{ }_{v}^{2 k} M\right)(z)=\bar{v}(M)(c z+d)^{-2 k} F(M z),
$$

where $M=\left(\begin{array}{ll}a & b \\ c & d\end{array}\right) \in G(\lambda)$ and $z \in \mathcal{H}$, then (11) and (12) become

$$
\left.F\right|_{v} ^{2 k} S_{\lambda}=F \quad \text { and }\left.\quad F\right|_{v} ^{2 k} T=F+q,
$$

respectively. It can be shown easily that the consistency condition (4) for a multiplier system $v$ in weight $2 k$ for $G(\lambda)$ is equivalent to

$$
\left.F\right|_{v} ^{2 k} M_{1} M_{2}=\left.\left(\left.F\right|_{v} ^{2 k} M_{1}\right)\right|_{v} ^{2 k} M_{2},
$$

where $M_{1}, M_{2} \in G(\lambda)$. From now on we shall write $F \mid M$ for $\left.F\right|_{v} ^{2 k} M$.

It is customary to denote by $q_{M}$ the period function associated with $M \in G(\lambda)$. Thus $F \mid M=F+q_{M}$ and from (16) we deduce that

$$
q_{M_{1} M_{2}}=q_{M_{1}} \mid M_{2}+q_{M_{2}} .
$$

Clearly, $q_{I}=q_{-I} \equiv 0$. When $\lambda$ is given by $(5)$, we have $\left(S_{\lambda} T\right)^{p}=(-1)^{r} I$. (See (6).) Repeated application of (17) yields

$$
q+q\left|\left(S_{\lambda} T\right)+q\right|\left(S_{\lambda} T\right)^{2}+\cdots+q \mid\left(S_{\lambda} T\right)^{p-1}=0 .
$$

From $T^{2}=-I$, we also conclude that

$$
q+q \mid(T)=0 .
$$




\section{THE CASE $\theta$ RATIONAL}

In this section we consider the case when $\lambda=2 \cos (\pi r / p)$ where $r$ and $p$ are relatively prime integers and $2 \leq r<p / 2$. As noted above, the group $G(\lambda)$ is not discrete, in this case. We shall make use of (18) and (19) to determine the LPPF's of positive weight for these groups. In what follows we shall assume that $k>0$ and $q$ is an LPPF of weight $2 k$ and multiplier system $v$ for the Hecke group $G(\lambda)$, where $\lambda$ is given by (5). Define

$$
M_{n}=V_{n} T=\left(S_{\lambda} T\right)^{n} T=\left(\begin{array}{ll}
e_{n} & f_{n} \\
g_{n} & h_{n}
\end{array}\right) .
$$

Combining (18) and (19), we obtain

$$
q\left|M_{p-1}+q\right| M_{p-2}+\cdots+q \mid M_{1}-q=0 .
$$

From ( 7 ) we see that $f_{n} \neq 0$ and $h_{n} \neq 0$ for $n=1, \ldots, p-2$. Thus we have

$$
\lim _{z \rightarrow 0}\left(q \mid M_{n}\right)(z)=\lim _{z \rightarrow 0} \bar{v}\left(M_{n}\right)\left(g_{n} z+h_{n}\right)^{-2 k} q\left(\frac{e_{n} z+f_{n}}{g_{n} z+h_{n}}\right)=L_{n},
$$

where

$$
L_{n}=\bar{v}\left(M_{n}\right) h_{n}^{-2 k} q\left(\frac{f_{n}}{g_{n}}\right), n=1, \ldots, p-2 .
$$

Combining (20) and (21), we get

$$
\lim _{z \rightarrow 0}\left(q \mid M_{p-1}-q\right)(z)=L,
$$

where

$$
L=-\sum_{n=1}^{p-2} L_{n}=-\sum_{n=1}^{p-2} \bar{v}\left(M_{n}\right) d_{n}^{-2 k} q\left(\frac{b_{n}}{d_{n}}\right) .
$$

Since $M_{p-1}=\left(S_{\lambda} T\right)^{p-1} T=T S_{\lambda}^{-1} T=\left(\begin{array}{cc}-1 & 0 \\ -\lambda & -1\end{array}\right)$, and since by consistency condition (4) for $v$ we have

$$
\bar{v}\left(T S_{\lambda}^{-1} T\right)(-\lambda z-1)^{-2 k}=\bar{v}\left(-T S_{\lambda}^{-1} T\right)(\lambda z+1)^{-2 k}=v\left(S_{\lambda}\right)(\lambda z+1)^{-2 k}
$$

(where in the last equality we have used Remark $2.1($ iii $)(c)$ ), we see that

$$
\left(q \mid M_{p-1}\right)(z)=v\left(S_{\lambda}\right)(\lambda z+1)^{-2 k} q\left(\frac{z}{\lambda z+1}\right) .
$$

Consequently,

$$
\lim _{z \rightarrow 0}\left\{v\left(S_{\lambda}\right)(\lambda z+1)^{-2 k} q\left(\frac{z}{\lambda z+1}\right)-q(z)\right\}=L .
$$

Replacing $z$ by $1 / z$ in the last limit yields

$$
\lim _{z \rightarrow \infty}\left\{v\left(S_{\lambda}\right)\left(\frac{z+\lambda}{z}\right)^{-2 k} q\left(\frac{1}{z+\lambda}\right)-q\left(\frac{1}{z}\right)\right\}=L .
$$

Taking the limit as $z \rightarrow \infty$ in (20) gives

$$
\lim _{z \rightarrow \infty}\left\{\bar{v}\left(S_{\lambda}\right) q(z+\lambda)-q(z)\right\}=0 .
$$


We now rewrite $q$ as:

$$
q(z)=\sum_{j=1}^{N} \sum_{t=0}^{m_{j}} a(j, t) z^{-\beta_{j}}(\log z)^{t}+\sum_{l=1}^{M} \sum_{t=0}^{n_{l}} b(l, t) z^{\alpha_{l}}(\log z)^{t}
$$

where

(26) $0 \leq \operatorname{Re}\left(\beta_{1}\right) \leq \cdots \leq \operatorname{Re}\left(\beta_{N}\right) ; m_{j} \leq m_{l}$ if $\operatorname{Re}\left(\beta_{j}\right)=\operatorname{Re}\left(\beta_{l}\right) \quad(j<l)$;

$$
0<\operatorname{Re}\left(\alpha_{1}\right) \leq \cdots \leq \operatorname{Re}\left(\alpha_{M}\right) ; n_{j} \leq n_{l} \text { if } \operatorname{Re}\left(\alpha_{j}\right)=\operatorname{Re}\left(\alpha_{l}\right) \quad(j<l)
$$

and introduce the following notations for further investigation of the limits in (23) and (24).

Let $z, \alpha \in \mathbf{C}, z \neq 0, z \neq-\lambda$, and $\nu \in \mathbf{Z}$. Then we define

$$
\begin{aligned}
& \phi_{\alpha, \nu}(z)=C_{1}(z+\lambda)^{\alpha}(\log (z+\lambda))^{\nu}-z^{\alpha}(\log z)^{\nu}, \\
& \psi_{\alpha, \nu}(z)=C_{2}\left(\frac{z+\lambda}{z}\right)^{-2 k}(z+\lambda)^{\alpha}(\log (z+\lambda))^{\nu}-z^{\alpha}(\log z)^{\nu} .
\end{aligned}
$$

In the above notations $C_{1} q(z+\lambda)-q(z)$ becomes

$$
\begin{aligned}
C_{1} q(z+\lambda)-q(z) & =\sum_{j=1}^{N} \sum_{t=0}^{m_{j}} a(j, t) \phi_{-\beta_{j}, t}(z)+\sum_{l=1}^{M} \sum_{t=0}^{n_{l}} b(l, t) \phi_{\alpha_{l}, t}(z) \\
& =\phi_{\alpha_{M}, n_{M}}(z) F(z),
\end{aligned}
$$

where $C_{1}=\bar{v}\left(S_{\lambda}\right)=e^{-2 \pi i \kappa}$ and

$$
F(z)=\sum_{j=1}^{N} \sum_{t=0}^{m_{j}} a(j, t) \frac{\phi_{-\beta_{j}, t}(z)}{\phi_{\alpha_{M}, n_{M}}(z)}+\sum_{l=1}^{M} \sum_{t=0}^{n_{l}} b(l, t) \frac{\phi_{\alpha_{l}, t}(z)}{\phi_{\alpha_{M}, n_{M}}(z)} .
$$

Similarly,

$$
\begin{aligned}
& C_{2}\left(\frac{z+\lambda}{z}\right)^{-2 k} q\left(\frac{1}{z+\lambda}\right)-q\left(\frac{1}{z}\right) \\
& =\sum_{j=1}^{N} \sum_{t=0}^{m_{j}} a(j, t)(-1)^{t} \psi_{\beta_{j}, t}(z)+\sum_{l=1}^{M} \sum_{t=0}^{n_{l}} b(l, t)(-1)^{t} \psi_{-\alpha_{l}, t}(z) \\
& =\psi_{\beta_{N}, m_{N}}(z) G(z),
\end{aligned}
$$

where $C_{2}=\bar{v}\left(-T S_{\lambda}^{-1} T\right)=e^{2 \pi i \kappa}$ and

$$
G(z)=\sum_{j=1}^{N} \sum_{t=0}^{m_{j}} a(j, t)(-1)^{t} \frac{\psi_{\beta_{j}, t}(z)}{\psi_{\beta_{N}, m_{N}}(z)}+\sum_{l=1}^{M} \sum_{t=0}^{n_{l}} b(l, t)(-1)^{t} \frac{\psi_{-\alpha_{l}, t}(z)}{\psi_{\beta_{N}, m_{N}}(z)} .
$$

We record (23) and (24) in

Proposition 3.1. If $q$ is an LPPF of weight $2 k$ and multiplier system $v$ for $G(\lambda)$, given by $(25)$ and $(27)$, then

$$
\lim _{z \rightarrow \infty} \phi_{\alpha_{M}, n_{M}}(z) F(z)=0 \quad \text { and } \lim _{z \rightarrow \infty} \psi_{\beta_{N}, m_{N}}(z) G(z)=L,
$$

where $F$ and $G$ are given by (27) and (28), respectively, and $L$ is as in (22). 
In [2], we have proved that $\lim _{z \rightarrow \infty} F(z) \neq 0$ and $\lim _{z \rightarrow \infty} G(z) \neq 0$ or the limits do not exist. Thus, there are sequences $\left\{z_{\nu}\right\}$ and $\left\{w_{\mu}\right\}$ such that

$$
\left|F\left(z_{\nu}\right)\right| \geq \delta \text { and }\left|G\left(w_{\mu}\right)\right| \geq \delta,
$$

for some positive real number $\delta$. This, together with (29), implies that

$$
\lim _{\nu \rightarrow \infty} \phi_{\alpha_{M}, n_{M}}\left(z_{\nu}\right)=0 \text { and }\left\{\psi_{\beta_{N}, m_{N}}\left(w_{\mu}\right)\right\} \text { is bounded. }
$$

The following theorem is a restatement of Theorem 3.1 of [2] and therefore the proof is omitted.

Theorem 3.1. Suppose $\lambda=2 \cos (\pi r / p)$, with $2 \leq r<p / 2,(r, p)=1$. Let $q$ be an LPPF of weight $2 k, k>0$, and multiplier system $v$ for the group $G(\lambda)$. Also let $v\left(S_{\lambda}\right)=e^{2 \pi i \kappa}$. We conclude:

(a) If $\kappa>0$, then $q \equiv 0$.

(b) If $\kappa=0$ and $k \geq 1$, then $q(z)=a\left(1-\bar{v}(T) z^{-2 k}\right)+b z^{-1}$, where $b=0$ if $k>1$.

Remark 3.1. In his first two papers on rational period functions [4] and [5], Knopp has shown that for the full modular group $\Gamma(1)$ the rational functions given by

$$
q(z)= \begin{cases}a\left(1-\bar{v}(T) z^{-2 k}\right), & \text { if } k>1, \\ a\left(1-\bar{v}(T) z^{-2}\right)+b z^{-1}, & \text { if } k=1\end{cases}
$$

are rational period functions. Here we are assuming that $k \in \mathbf{Z}$. In fact he showed that these are the only rational period functions with rational poles. It can easily be seen that the functions given above are period functions for Hecke groups $G(\lambda)$.

We are now in a position to state and prove our main results.

Theorem 3.2. Suppose $\lambda=2 \cos (\pi r / p)$, with $2 \leq r<p / 2, \quad(r, p)=1$. If $\kappa>0$, then there is no nontrivial automorphic integral of positive weight for the Hecke group $G(\lambda)$.

Proof. For if there is an automorphic integral $F$ of weight $2 k, k>0$, then by Theorem $3.1(a)$, its LPPF $q$ is given by $q \equiv 0$. But then, $F$ would be an automorphic form of weight $2 k, k>0$, and so must be identically zero, since the group is nondiscrete.

Theorem 3.3. Suppose $\lambda=2 \cos (\pi r / p)$, with $2 \leq r<p / 2, \quad(r, p)=1$. If $\kappa=0$, then there is no nontrivial automorphic integral of weight $2 k, k \geq 1$, for the Hecke group $G(\lambda)$.

Proof. First suppose $k>1$. In this case the LPPF of an automorphic integral $F$ is given by $q(z)=a\left(1-\bar{v}(T) z^{-2 k}\right)$. Define $G(z)=F(z)+a$. Then, since $\kappa=0$, we have

$$
\left(G \mid S_{\lambda}\right)(z)=\left(F \mid S_{\lambda}\right)(z)+\left(a \mid S_{\lambda}\right)(z)=F(z)+a=G(z)
$$

and

$$
\begin{aligned}
(G \mid T)(z) & =(F \mid T)(z)+(a \mid T)(z)=F(z)+q(z)+a \bar{v}(T) z^{-2 k} \\
& =F(z)+a\left(1-\bar{v}(T) z^{-2 k}\right)+a \bar{v}(T) z^{-2 k}=F(z)+a=G .
\end{aligned}
$$

Thus we have an automorphic form $G$ of positive weight. Once again $G$ has to be identically zero, thereby forcing $F$ to be a constant, i.e., $F \equiv-a$. 
Next suppose $k=1$ and assume that there is an automorphic integral $F$ of weight 2. First observe that $\bar{v}(T)= \pm 1$ (see Remark 2.1(iii)). Thus we may assume that $\bar{v}(T)=1$. The LPPF of $F$ is given by $q(z)=a\left(1-z^{-2}\right)+b z^{-1}$. Put $G=F+a$. Then $G$ is an automorphic integral of weight 2 with $\operatorname{LPPF} p(z)=b z^{-1}$. If $b=0$, then $G$ is an automorphic form, and hence it is identically zero. Thus $F$ is a constant. If $b \neq 0$, then we divide $G$ by $-b$ to get an automorphic integral with period function $\frac{-1}{z}$. Thus we may assume that $F$ is an automorphic integral with LPPF $q$ given by $q(z)=\frac{-1}{z}$. Define

$$
H(z)=\int_{i}^{z} F(\tau) d \tau
$$

Since $F$ has an exponential series expansion of the form $F(\tau)=\sum_{n=0}^{\infty} a_{n} e^{2 \pi i n \tau / \lambda}$, we see that

$$
H(z)=a_{0} z+\frac{\lambda}{2 \pi i} \sum_{n=1}^{\infty} \frac{a_{n}}{n} e^{2 \pi i n z / \lambda}+C
$$

where $C$ is a constant. Thus $H(z+\lambda)=H(z)+a_{0} \lambda$. By a simple change of variable (from $\tau$ to $-1 / \tau$ ), we can easily see that

$$
H\left(\frac{-1}{z}\right)=H(z)-\log z+\frac{\pi i}{2} .
$$

Now define $I(z)=e^{H(z)}$. Then

$$
I(z+\lambda)=\rho I(z) \text { and } I\left(\frac{-1}{z}\right)=e^{\pi i / 2} z^{-1} I(z),
$$

where $\rho=e^{a_{0} \lambda}$. Put

$$
K(z)=\frac{I(z)}{I^{\prime \prime}(z)}
$$

Then $K(z+\lambda)=K(z)$ and $K\left(\frac{-1}{z}\right)=z^{-1} K(z)$. Thus $K$ is an automorphic form of weight -1 . But this is not possible. This completes the proof.

\section{Aknowledgment}

I would like to thank Marvin Knopp for introducing me to the subject of Analytic Number Theory and suggesting this problem. I would also like to thank the referee for many valuable comments and suggestions.

\section{REFERENCES}

[1] Evans, Ronald. 1973. A Fundamental Region for Hecke's Modular Groups. J. of Number Theory 5: 108-115. MR 47:3319

[2] Hassen, Abdulkadir, 1997. Log-Polynomial Period Functions for Hecke Groups. The Ranamujan Journal. To appear.

[3] Hecke, Erich. 1938. Lectures on Dirichlet series, modular functions and quadratic forms. Ann Arbor: Edwards Brothers.

[4] Knopp, Marvin. 1978. Rational period functions of the modular group. Duke Math. J. 45: 47-62. MR 58:5522

[5] Knopp, Marvin. 1981. Rational period functions of the modular group II. Glasgow Math. J. 22: 185-197. MR 83g:10018

[6] Knopp, Marvin. 1994. On Dirichlet series satisfying Riemann's functional equation. Invent. Math. 117 (1994): 361-372. MR 95h:11087

[7] Knopp, Marvin, and Sheingorn, Mark. 1996. Dirichlet series and Hecke triangle groups of infinite volume. Acta. Arith., 76 (1996): 227-244. MR 97h:11054 
[8] Siegel, C. Ludwig. 1949. Transcendental Numbers. Anal. of Math. Stud. Princeton University Press. MR 11:330c

[9] Weil, André. 1977. Some Remarks on Hecke's Lemma and Its Use. In: Alg. Numb. Th., International Symposium, Kyoto, Japan. MR 58:699

Department of Mathematics, Rowan University, Glassboro, New Jersey 08028

E-mail address: hassen@rowan.edu 